\title{
Circulating vascular endothelial growth factor receptor 2 levels and their association with lipid abnormalities in patients on hemodialysis
}

\author{
LESZEK NIEPOLSKI $^{1}$, HANNA DRZEWIECKA $^{2}$ and WOJCIECH WARCHOє ${ }^{3}$ \\ Departments of ${ }^{1}$ Physiology, ${ }^{2}$ Biochemistry and Molecular Biology, and ${ }^{3}$ Ophthalmology and Optometry, \\ Poznan University of Medical Sciences, Poznań 60-781, Poland
}

Received May 10, 2020; Accepted February 8, 2021

DOI: $10.3892 / b r .2021 .1413$

\begin{abstract}
The aim of the present study was to examine the association between the levels of circulating vascular endothelial growth factor receptor (VEGFR)2 levels, serum lipid composition and plasma receptor for advanced glycation end-products (RAGE) expression in patients undergoing hemodialysis (HD). A total of 50 patients on HD (27 men and 23 women; median age, 66 years; age range $28-88$ years; HD mean time, 29.0, 3.9-157.0 months) were enrolled. Age-matched healthy subjects $(n=26)$ were used as the control group. Plasma VEGFR2 and RAGE levels were determined using ELISA. Dyslipidemia (D) in patients on HD was diagnosed according to the Kidney Disease Outcomes Quality Initiative Clinical Practice Guidelines for Managing Dyslipidemias in Chronic Kidney Disease. Circulating VEGFR2, RAGE and serum lipids were compared between dyslipidemic and non-dyslipidemic patients on HD and controls. In patients on HD, the plasma VEGFR2 levels were lower compared with those in the healthy population. D was associated with high plasma VEGFR2 levels. The triglyceride/HDL-cholesterol ratio was strongly associated with plasma VEGFR2 levels. The plasma
\end{abstract}

Correspondence to: Dr Leszek Niepolski, Department of Physiology, Poznan University of Medical Sciences, Święcickiego 6 Street, Poznań 60-781, Poland

E-mail: lesz.nie@tlen.pl

Abbreviations: AGE, advanced glycation end-products; BMI, body mass index; DBW, dry body weight; D, dyslipidemia; ESRD, end stage renal disease; HbAlc glycated haemoglobin; HD, hemodialysis; HDL, high-density lipoprotein; HDL-chol, HDL-cholesterol; HOMA-IR, homeostasis model assessment of insulin resistance; hsCRP, high sensitivity $\mathrm{C}$ reactive protein; K/DOQI, Kidney Disease Outcomes Quality Initiative; LDL, low-density lipoprotein; LDL-chol, LDL-cholesterol; MeS, metabolic syndrome; NAFLD, nonalcoholic fatty liver disease; RAGE, receptor for AGEs; Tchol, total cholesterol; TG, triglyceride; WC, waist circumference; WheR, waist to height ratio; VEGF, vascular endothelial growth factor; VEGFR, VEGF receptor

Key words: dyslipidemia, hemodialysis, receptor for advanced glycation end-products, vascular endothelial growth factor receptor 2
VEGFR2 concentration was associated with circulating RAGE levels. Therefore, circulating VEGFR2 levels may be partly associated with lipid abnormalities and plasma RAGE levels in patients receiving HD.

\section{Introduction}

Patients with end-stage renal disease (ESRD) on maintenance hemodialysis (HD) are susceptible to changes in their blood vessels. A number of studies have confirmed the high prevalence of atherosclerotic lesions in the arteries of patients on HD $(1,2)$. It is well established that the function of the endothelium is intricately involved in atherogenesis (3). ESRD leads to altered properties and responses of the endothelium. However, the mechanisms through which uremia affects endothelial cells and causes atherosclerotic changes remain elusive. Therefore, it is worth exploring alternative metabolic pathways that may be involved in atherosclerotic formation in patients on HD. Vascular endothelial growth factor (VEGF) and its receptors (VEGFRs) are crucial regulators of vasculogenesis and angiogenesis under physiological and pathophysiological conditions (3). The VEGF-VEGFR axis regulates several biological functions in the endothelium. Human endothelial cells express three related membrane-bound VEGFRs, VEGFR1, VEGFR2 and VEGFR3, which are encoded by different genes. All membrane-bound receptors possess an extracellular, transmembrane and an intracellular domain that mediates critical signalling pathways in endothelial cells (4). In addition, circulating isoforms are present in human plasma. Due to the alternative splicing of VEGFR mRNA, and also due to proteolytic shedding of the extracellular domain, membranous receptors may exist as soluble truncated forms (sVEGFR1, 2 and 3). These soluble forms are secreted by endothelial cells or are proteolytically cleaved $(5,6)$. The role of the splice variant sVEGFR-1 is well established (7), less is known regarding sVEGFR2 and sVEGFR3. It has been reported that the membrane-bound and spliced isoforms of VEGFR2 participate in several important pathophysiological processes, such as the production of vasoactive mediators involved in hypertension, thrombosis and inflammation (8-12), non-alcoholic fatty liver disease (NAFLD) $(13,14)$, production of nitric oxide (1) and prostacyclin (2). VEGFR2 is 
activated through ligand-stimulated receptor dimerization and phosphorylation (15). In addition, the dimerization and phosphorylation of VEGFR2 may be modulated by modifying the lipid raft of endothelial cell membranes through high-density and low-density lipoproteins (16-19), advanced glycation end-products (AGEs) and their receptors $(20,21)$. Therefore, metabolic abnormalities may lead to endothelial cell membrane VEGFR2 relocation and expression, conferring changes to the formation of circulating VEGFR2 isoforms. No clinical studies have assessed the association between plasma VEGFR2 concentrations, lipid abnormalities and receptor for AGE (RAGE) levels. Therefore, in the present pilot study, circulating (soluble truncated) VEGFR2 concentrations were examined in relation to lipid composition and plasma RAGE levels in patients on HD, and biochemical and anthropometric parameters were evaluated.

\section{Materials and methods}

Ethics statement. The protocol used in the present study was performed in accordance with the guidelines described in the Declaration of Helsinki (22) and was approved by the Institutional Review Board of Poznań University of Medical Sciences, Poland. Written informed consent was obtained from all subjects prior to participation.

Patients. A total of 50 Caucasian patients on HD (27 men and 23 women; median age, 66 years; age range, $28-88$ years; HD mean time, 29.0, 3.9-157.0 months) who had been treated with maintenance HD for $\geq 6$ months were recruited. All patients underwent HD 3 times for 4-4.5 h per week on low-flux polysulphone-based membranes with a surface area of $1.3-2.1 \mathrm{~m}^{2}$, low-molecular-weight heparin was used as an anticoagulant. The dialysis efficiency was evaluated based on single-pool $\mathrm{Kt} / \mathrm{V}$ urea nitrogen, according to the National Kidney Foundation-Kidney Disease Outcomes Quality Initiative (K/DOQI) Guidelines (23). Patients on HD who fell into one or more of the following categories were excluded: i) Suffered from a hepatic disease, neoplastic disease, active collagen disease, acute coronary syndrome and/or not well-controlled diabetes mellitus; ii) cerebral stroke in the 6 months preceding the commencement of the study; or iii) received statins or fibrates in the 12 weeks prior to the commencement of the present study. None of the patients were receiving antibiotics, corticosteroids or immunosuppressant drugs at the time of the study. According to the abdominal ultrasound examination, 10 of the patients on HD (20\%) also suffered from NAFLD. The patients were recruited between July and November 2018. The fasting blood samples were taken on December 3rd and 4th, 2018 (prior to the HD session) from patients at the Dialysis Center, B.Braun Avitum Poland, Nowy Tomyśl, Poland.

Controls. A total of 26 age-matched self-declared healthy Caucasian volunteers were included in the control group. No substantial health deviations were recorded during the medical interview and physical examinations. The volunteers had not received lipid-lowering drugs, at least in the 3 months prior to the commencement of the study. Control individuals were recruited between June and November 2018, and all controls were students from the University of the Third Age in Nowy
Tomyśl and Wolsztyn, Poland. The blood samples were taken on December 10th and 11th, 2018 (according to the protocol).

Clinical and laboratory methods. According to Kouw et al (24), dry body weight (DBW) was defined as the weight at the end of an HD session, when a patient was more susceptible to developing symptoms of hypotension. DBW was determined by an experienced nephrologist. Anthropometric parameters measured included body mass index (BMI), waist circumference (WC), waist-to-height ratio (WHeR) and waist-to-hip ratio (WHR). Weight was measured to the nearest $10 \mathrm{~g}$. Height was measured to the nearest $5 \mathrm{~mm}$, using a wall-mounted stadiometer. BMI $\left(\mathrm{kg} / \mathrm{m}^{2}\right)$ was calculated as DBW $(\mathrm{kg}) /$ height $(\mathrm{m})^{2}$. WC was measured midway between the lowest lateral border of the ribs and the uppermost lateral iliac crest. WHeR was calculated as WC/height. WHR was measured as WC/hip circumference, fasting blood samples were collected from each patient at the start of a midweek HD session from the arterial site of the arterio-venous fistula, catheter, or antecubital vein of the participants. NAFLD was diagnosed by abdominal ultrasonography using a method established by Hamaguchi et al (25), which included hepatorenal echo contrast, liver brightness, deep attenuation and vascular blurring.

Dyslipidaemia (D) in the control group was assessed according to The European Society of Cardiology and the European Atherosclerosis Society guidelines (26). D in patients on HD was diagnosed according to the K/DOQI Clinical Practice Guidelines for Managing Dyslipidemias in Chronic Kidney Disease (2003) (27). Patients diagnosed with D with a serum low-density cholesterol (LDL-chol) concentration $\geq 100 \mathrm{mg} / \mathrm{dl}$ were considered hyper-LDL cholesterolemic, whereas those with a non-high-density cholesterol (non-HDL-chol) levels of $\geq 130 \mathrm{mg} / \mathrm{dl}$ and triglycerides (TGs) levels of $\geq 200 \mathrm{mg} / \mathrm{dl}$ were considered hyper-TG/hyper-non-HDL cholesterolemic. Patients who met one of these criteria were included in the dyslipidemic group. The remaining patients were referred to as non-dyslipidemic, according to the K/DOQI criteria. To determine the atherogenic pattern of $\mathrm{D}$, the TG/HDL-chol ratio was used as the atherogenic index (AI). A ratio value $\geq 3.8$ was considered to indicate atherogenic D (27). HD subjects with a TG/HDL chol ratio value $<3.8$ were described as patients without atherogenic D (28). Total chol(Tchol; cat.no.03039773190),HDL-chol (cat. no. 04399803190) and TG (cat. no. 20767107322) were assessed using enzymatic and colorimetric methods, according to the manufacturer's protocol (Cobas Integra, Roche Diagnostics GmbH). The LDL-chol concentration was calculated using the Friedewald formula (29). In patients with serum TG concentrations of $\geq 400 \mathrm{mg} / \mathrm{dl}$, LDL-chol was measured directly (BioSystems S.A.). Non-HDL-chol was estimated as the Tchol minus HDL-chol. The LDL-chol was calculated using the Friedewald formula.

Plasma VEGFR2, RAGE, insulin, glycated hemoglobin (HbA1c), glucose levels, lipid profiles, albumin levels and high-sensitivity $\mathrm{C}$-reactive protein (hsCRP) concentration were measured. After a minimum of $8 \mathrm{~h}$ of overnight fasting, venous blood was drawn into an EDTA tube and promptly centrifuged at $2,000 \times \mathrm{g}$ for $10 \mathrm{~min}$ at $4^{\circ} \mathrm{C}$. The obtained plasma was frozen at $-80^{\circ} \mathrm{C}$ in aliquots until protein analysis was performed. The plasma VEGFR2 levels were measured 
Table I. Clinicopathological characteristics of the hemodialysis patients and controls with and without D.

\begin{tabular}{|c|c|c|c|c|c|c|}
\hline \multirow[b]{2}{*}{ Parameter } & \multicolumn{3}{|c|}{ HD patients, $n=50$} & \multicolumn{3}{|c|}{ Controls, $n=26$} \\
\hline & With D & Without D & P-value & With D & Without D & P-value \\
\hline $\mathrm{N}$ & 26 & 24 & - & 15 & 11 & - \\
\hline Age, years ${ }^{b}$ & $65,47-89$ & $66,28-81$ & 0.938 & $72,60-90$ & $68,61-77$ & 0.338 \\
\hline Sex & & & & & & - \\
\hline Male & 14 & 13 & & 6 & 5 & \\
\hline Female & 11 & 12 & & 9 & 6 & \\
\hline DM, n (\%) & $11(42)$ & $15(62)$ & - & 0 & 0 & - \\
\hline Waist to hip ratio ${ }^{a}$ & $0.965 \pm 0.095$ & $0.968 \pm 0.096$ & 0.933 & $0.918 \pm 0.042$ & $0.894 \pm 0.098$ & 0.067 \\
\hline Waist to height ratio ${ }^{a}$ & $0.615 \pm 0.084$ & $0.613 \pm 0.102$ & 0.931 & $0.596 \pm 0.076$ & $0.531 \pm 0.063$ & 0.642 \\
\hline $\mathrm{SBP}, \mathrm{mmHg}^{\mathrm{a}}$ & $123 \pm 15$ & $125 \pm 15$ & 0.598 & $130 \pm 10$ & $128 \pm 10$ & 0.743 \\
\hline $\mathrm{DBP}, \mathrm{mmHg}^{\mathrm{b}}$ & $70,60-90$ & $70,60-80$ & 0.825 & $80,60-90$ & $70,60-100$ & 0.442 \\
\hline $\mathrm{BMI}, \mathrm{kg} / \mathrm{m} 2^{\mathrm{a}}$ & $26.6 \pm 4.8$ & $27.1 \pm 5.9$ & 0.742 & $27.7 \pm 5.42$ & $27.3 \pm 3.44$ & 0.723 \\
\hline Period of HD, months ${ }^{\mathrm{a}}$ & $32.2 \pm 20.8$ & $41 \pm 36.1$ & 0.641 & - & - & - \\
\hline $\mathrm{eKT} / \mathrm{V}^{\mathrm{a}}$ & $1.34 \pm 0.23$ & $1.36 \pm 0.18$ & 0.675 & - & - & - \\
\hline Residual diuresis, $1 / 24 \mathrm{~h}$, range & $0.5,0-2.5$ & $0.6,0-2.1$ & 0.754 & - & - & - \\
\hline
\end{tabular}

${ }^{a}$ Mean \pm standard deviation; ${ }^{b}$ median, range. BMI, body mass index; DBP, diastolic blood pressure; DM, diabetes mellitus; D, dyslipidemia; $\mathrm{HD}$, hemodialysis; eKt/V, adequacy of HD; SBP, systolic blood pressure; DBP, diastolic blood pressure.

using the Human VEGFR2 ELISA kit (cat. no. ab213476; Abcam), according to the manufacturer's protocols. No significant cross-reactivity or interference was observed. The limits of VEGFR2 concentration detection were 34.3-25,000 pg/ml. The sensitivity of the assay was $<70 \mathrm{pg} / \mathrm{ml}$. The intra-assay coefficient of variation $(\mathrm{CV})$ was $2.5 \%$, and the $\mathrm{CV}$ for inter-assay precision was $5.8 \%$. The plasma RAGE levels were measured using the Human RAGE ELISA kit (cat. no. ab190807; Abcam). All measurements were performed in duplicate. Plasma insulin concentrations were determined using the electrochemiluminescence immunoassay method, according to the manufacturer's protocol (Cobas E411; cat. no. 12017547122; Roche Diagnostics GmbH). Homeostasis model assessment of insulin resistance (HOMA-IR) was determined as fasting plasma insulin $(\mu \mathrm{U} / \mathrm{ml}) \times$ fasting plasma glucose $(\mathrm{mmol} / \mathrm{l}) / 22.5$ (30). HbAlc was determined using the turbidimetric inhibition immunoassay method (Cobas Integra; cat. no. 04528123 190; Roche Diagnostics GmbH). The concentration of hsCRP was determined using a high-sensitivity latex enhanced immunoturbidimetry method, according to the manufacturer's protocol (Cobas Integra; cat. no. 04628918190; Roche Diagnostics $\mathrm{GmbH}$ ). Other biochemical parameters were measured using standard laboratory techniques with a certified automatic analyzer (Cobas Integra 400; Roche Diagnostics Ltd.).

Categorization of patients. Participants were categorized as having $\mathrm{D}$ when they met the criteria described in the K/DOQI for D (29). Metabolic syndrome (MeS) was diagnosed according to the International Diabetes Federation Worldwide Definition (31). Selected groups were compared to each other and analysed separately. In order to identify potential predictors of plasma VEGFR2 levels, the statistical analysis was performed on the entire HD group.
Statistical analysis. The normality of distribution of variables was assessed using the Shapiro-Wilk test for each group separately. Numeric variables are expressed as the mean \pm standard deviation, or as a median and range, as appropriate; categorical variables are presented as percentages. A Student's t-test was used to compare normally distributed data, or otherwise a Mann-Whitney U test was used. As plasma VEGFR2 levels were not normally distributed, a Spearman's rank correlation was performed to determine the correlation between this variable and the other parameters. Univariate receiver operating characteristic (ROC) curves were evaluated using Medical kit version 4.0.67 (statsoft.pl). Statistical analysis was performed using STATISTICA version 13 (TIBCO Software Inc.). $\mathrm{P}<0.05$ was considered to indicate a statistically significant difference.

\section{Results}

Comparison between the entire HD group and control subjects. The clinical and demographic characteristics of patients in the HD and control groups, with and without D, are summarized in Table I. Despite the prior declaration of good health, $57 \%$ of the subjects in the control group had D, with a similar percentage observed in the HD group (54\%). The HD subjects had a higher TG/HDL-chol ratio, as compared with age-matched controls (4.7 vs. 1.28; $\mathrm{P}<0.0005$; Table II). The AI in the HD group was $56 \%$, whereas there were no subjects with atherogenic $\mathrm{D}$ in the control group. A higher prevalence of MeS was recorded in patients on HD compared with the control subjects ( 60 vs. $15 \%$, respectively; $\mathrm{P}=0.001$ ). The HD group had a similar percentage of cigarette smokers, BMI and WHeR to that of controls, but a higher WHR ratio $(\mathrm{P}=0.006)$. Compared with the control, the entire HD group exhibited a lower plasma VEGFR2 concentration ( $\mathrm{P}=0.025$; Fig. 1$)$, Tchol, LDL-chol, HDL-chol and HDL/Tchol ratio. Additionally, patients on HD 
Table II. Values of laboratory parameters in HD patients and controls.

\begin{tabular}{|c|c|c|c|}
\hline Parameter & HD patients, $n=50$ & Controls, $n=26$ & P-value \\
\hline VEGFR2, ng/ml ${ }^{\mathrm{e}}$ & $1.32,0.330-5.0$ & $2.24,0.700-5.9$ & $0.025^{\mathrm{a}}$ \\
\hline Albumin, $\mathrm{g} / \mathrm{dl}^{\mathrm{d}}$ & $3.65 \pm 0.33$ & $4.32 \pm 0.21 .0$ & 0.054 \\
\hline Total cholesterol, $\mathrm{mg} / \mathrm{dl}^{\mathrm{d}}$ & $175.0 \pm 49.0$ & $203.0 \pm 43.0$ & $0.001^{\mathrm{b}}$ \\
\hline LDL-cholesterol, mg/dl ${ }^{\mathrm{d}}$ & $93.3 \pm 33.2$ & $115.0 \pm 36.6$ & $0.005^{\mathrm{b}}$ \\
\hline HDL-cholesterol, mg/dl ${ }^{\mathrm{d}}$ & $41.8 \pm 12.0$ & $68.3 \pm 19.2$ & $0.005^{\mathrm{b}}$ \\
\hline $\mathrm{TG}, \mathrm{mg} / \mathrm{dl}^{\mathrm{d}}$ & $173.0 \pm 83.0$ & $94.0 \pm 33.0$ & $<0.0001^{\mathrm{c}}$ \\
\hline Non-HDL, mg/dl ${ }^{\mathrm{d}}$ & $133.0 \pm 47.0$ & $137.0 \pm 38$ & 0.676 \\
\hline LDL/HDL-cholesterol ratio ${ }^{\mathrm{d}}$ & $2.35 \pm 0.87$ & $1.70 \pm 0.64$ & $0.004^{\mathrm{b}}$ \\
\hline HDL/total cholesterol, \% ${ }^{\mathrm{e}}$ & $22.2,14-50$ & $32.9,55-21$ & $0.0005^{\mathrm{c}}$ \\
\hline TG/HDL-cholesterol ratio ${ }^{\mathrm{e}}$ & $4.17,0.8-8.2$ & $1.28,0.5-3.5$ & $<0.0005^{\mathrm{c}}$ \\
\hline hsCRP, mg/ld & $14.1 \pm 2.6$ & $5.0 \pm 1.68$ & 0.05 \\
\hline RAGE $n g / \mathrm{ml}^{\mathrm{d}}$ & $1.23 \pm 1.06$ & $0.680 \pm 0.048$ & $0.035^{\mathrm{a}}$ \\
\hline $\mathrm{HB}, \mathrm{g} / \mathrm{dl}^{\mathrm{d}}$ & $11.4 \pm 1.4$ & $13.5 \pm 0.9$ & 0.07 \\
\hline
\end{tabular}

${ }^{\mathrm{a}} \mathrm{P}<0.05$; ${ }^{\mathrm{b}}<0.01 ;{ }^{\mathrm{c}} \mathrm{P}<0.001$; ${ }^{\mathrm{d}}$ mean \pm standard deviation; ${ }^{\mathrm{e}}$ median, range. HB, hemoglobin; HD, hemodialysis; HDL, high density lipoprotein; hsCRP, high sensitivity C reactive protein; LDL, low density lipoprotein; RAGE, receptor for advanced glycation end products; TG, triglyceride; VEGFR2, vascular endothelial growth factor receptor 2.

Table III. Laboratory parameters in HD patients with and without dyslipidemia.

\begin{tabular}{|c|c|c|c|}
\hline Parameter & HD with D & HD without D & P-value \\
\hline VEGFR2, ng/ml ${ }^{\mathrm{d}}$ & $1.96,0.330-5.0$ & $0.930,0.390-3.31$ & $0.01^{\mathrm{a}}$ \\
\hline Albumin, $\mathrm{g} / \mathrm{dl}^{\mathrm{c}}$ & $3.74 \pm 0.32$ & $3.56 \pm 0.32$ & 0.058 \\
\hline Total cholesterol, $\mathrm{mg} / \mathrm{dl}^{\mathrm{d}}$ & $194.0,159.0-349.0$ & $136.0,98.0-178.0$ & $<0.0001^{\mathrm{b}}$ \\
\hline LDL-cholesterol, $\mathrm{mg} / \mathrm{dl}^{\mathrm{c}}$ & $118.0 \pm 22.3$ & $66.3 \pm 18.3$ & $<0.0001^{\mathrm{b}}$ \\
\hline HDL-cholesterol, mg/dl ${ }^{\mathrm{d}}$ & $38.0,27.0-67.0$ & $38.5,26.0-82.0$ & 0.953 \\
\hline $\mathrm{TG}, \mathrm{mg} / \mathrm{dl}^{\mathrm{c}}$ & $220.0 \pm 82.9$ & $122.0 \pm 44.5$ & $<0.0001^{\mathrm{b}}$ \\
\hline Non-HDL, mg/dl ${ }^{\mathrm{d}}$ & $156.0,127.0-300.0$ & $94.5,58.0-139$ & $<0.0001^{\mathrm{b}}$ \\
\hline LDL/HDL-cholesterol ratio ${ }^{c}$ & $2.95 \pm 0.62$ & $1.69 \pm 0.62$ & $<0.0001^{\mathrm{b}}$ \\
\hline HDL/Total cholesterol, $\%^{\mathrm{d}}$ & $19.0,14.0-33.5$ & $30.2,17.3-50.0$ & $<0.0001^{\mathrm{b}}$ \\
\hline TG/HDL-cholesterol ratio ${ }^{\mathrm{d}}$ & $5.2,1.82-10.1$ & $2.76,0.810-7.08$ & $0.0005^{b}$ \\
\hline hsCRP, mg/l ${ }^{\mathrm{d}}$ & $4.7,4.0-126.0$ & $9.45,4.0-65.1$ & 0.41 \\
\hline $\mathrm{HB}, \mathrm{g} / \mathrm{dl}^{\mathrm{c}}$ & $11.3 \pm 1.4$ & $11.4 \pm 1.4$ & 0.772 \\
\hline
\end{tabular}

${ }^{\mathrm{a}} \mathrm{P}<0.01 ;{ }^{\mathrm{b}} \mathrm{P}<0.001 ;{ }^{\mathrm{c}}$ mean \pm standard deviation; ${ }^{\mathrm{d}}$ median, range. D, dyslipidemia; HB, hemoglobin; HD, hemodialysis; HDL, high density lipoprotein; hsCRP, high sensitivity $\mathrm{C}$ reactive protein; LDL, low density lipoprotein; RAGE, receptor for advanced glycation end products; TG, triglyceride; VEGFR2, vascular endothelial growth factor receptor 2.

exhibited a significantly elevated serum TG, LDL/HDL-chol ratio, TG/HDL-chol ratio and plasma RAGE levels.

Comparison of VEGFR2 levels between patients on HD with and without $D$. The laboratory parameters of patients on HD with and without D are presented in Table III. In patients on HD with D, the plasma VEGFR2 and serum Tchol, TG, HDL-chol, LDL-chol, non-HDL-chol levels, as well as the LDL-/HDL-chol and TG/HDL-chol ratios, were higher compared with those in patients on HD without D. The patients on HD with D had a significantly higher AI, compared with patients on HD without D (73 vs. 47\%, respectively). In addition, the receiver operating characteristic (ROC) curve analysis revealed that a plasma VEGFR2 level of 1.33 was the ideal cut-off value for the prognosis of patients on HD with and without D [area under the ROC curve, $0.713 ; 95 \%$ confidence interval (CI): 0.569-0.858; Fig. 2].

Comparison of VEGFR2 levels in the control group with and without $D$. The laboratory parameters of controls with and without D are presented in Table IV. Control subjects with D did not differ significantly from those without $\mathrm{D}$ with respect to plasma VEGFR2, serum HDL-chol and TG concentrations. In the control subjects with D, the serum Tchol, LDL-chol and non-HDL-chol levels, as well as LDL-/HDL-chol ratio, were higher compared with those in control subjects without D. 


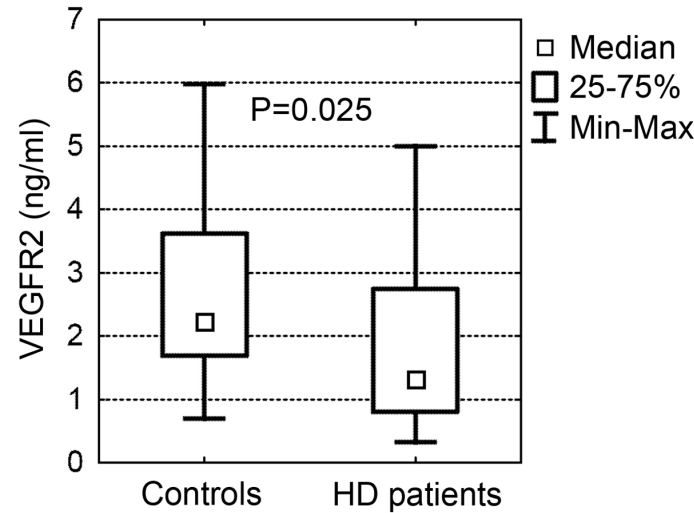

Figure 1. Comparison of plasma VEGFR2 concentration in HD patients and controls. VEGFR2, vascular endothelial growth factor receptor 2; HD, hemodialysis.

VEGFR2 ROC curve Criterion, VEGFR2 $>1.33(\mathrm{ng} / \mathrm{ml})$ Sensitivity, 0.692; Specificity, 0.708

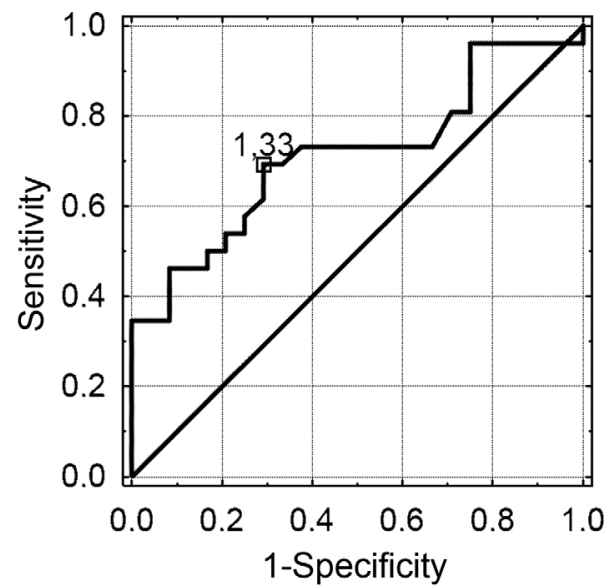

Figure 2. ROC curve of VEGFR2 in the prognosis of dyslipidemia in patients receiving hemodialysis. VEGFR2, vascular endothelial growth factor receptor 2 ; ROC, receiver operating characteristic.

Comparison of VEGFR2 levels between patients on $H D$ with and without MeS. There was no difference in plasma VEGFR2 levels between patients with and those without MeS $(1.38 \pm 0.915$ vs. $1.10 \pm 0.785 \mathrm{ng} / \mathrm{ml}$, respectively; $\mathrm{P}=0.338)$.

Correlation analysis of VEGFR2 levels and other parameters in patients on $H D$ and controls. In the control group, plasma VEGFR2 levels were significantly positively correlated with TG/HDL-chol ratio and TG levels (Table V). In the entire HD group, there were positive correlations between plasma VEGFR2 levels and age, RAGE (Fig. 3), Tchol, LDL, TG, non-HDL, LDL/HDL-chol ratio and TG/HDL-chol ratio (Fig. 4), and a negative correlation between plasma VEGFR2 levels and HDL/Tchol ratio. Of note, there was no correlation between plasma VEGFR2 levels and residual diuresis, HOMA-IR, hsCRP or anthropometric parameters in any of the examined groups. In the HD group with D, there were positive correlations between the plasma levels of VEGFR2 with age and RAGE. Similarly, in the group without D, significant positive correlations were observed between plasma

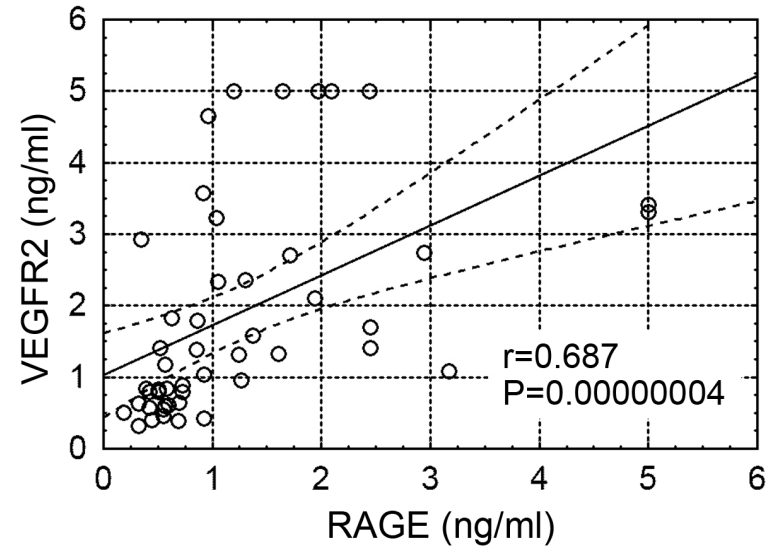

Figure 3. Correlation between plasma VEGFR2 and plasma RAGE expression levels in the entire hemodialysis group. VEGFR2, vascular endothelial growth factor receptor 2; RAGE, receptor for advanced glycation end-products.

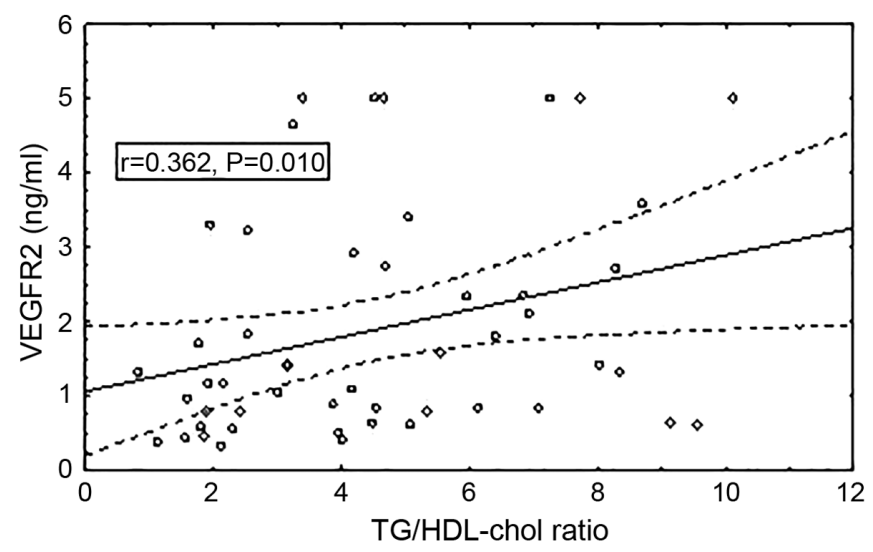

Figure 4. Correlation between plasma VEGFR2 and TG/HDL-chol levels in the entire hemodialysis group. VEGFR2, vascular endothelial growth factor receptor 2; TG, triglycerides; HDL-chol, high density lipoprotein cholesterol.

VEGFR2 and RAGE levels, whereas a significant negative correlation was observed between plasma VEGFR2 levels and platelet counts. There was no correlation between plasma VEGFR2 levels and the presence of NAFLD $(\mathrm{P}=0.818)$ based on the abdominal ultrasound examination of patients on HD. The significant correlations of plasma VEGFR2 levels in the examined groups are presented in Table V.

\section{Discussion}

To the best of our knowledge, there are no previous studies showing the association of plasma VEGFR2 levels with lipid abnormalities, MeS, plasma RAGE levels and other biochemical parameters in patients on HD. The following were the major findings of the present study in patients on HD: i) The plasma VEGFR2 levels were lower compared with those in the control subjects; ii) in patients on HD with D, the plasma VEGFR2 levels were higher compared with those in patients on HD without D; iii) there were positive correlations between plasma VEGFR2 levels and lipid abnormalities (Tchol, LDL, TG, Non-HDL, LDL/HDL-chol ratio, TG/HDL-chol ratio and plasma RAGE levels); iv) there was no difference in plasma VEGFR2 levels between patients with and without MeS. 
Table IV. Laboratory parameters in the control cohort with and without D.

\begin{tabular}{lccc}
\hline Parameter $^{\text {b }}$ & Controls with D & Controls without D & P-value \\
\hline VEGFR2, ng/ml & $2.75 \pm 1.63$ & $2.39 \pm 1.14$ & 0.535 \\
Albumin, g/dl & $4.31 \pm 0.18$ & $4.34 \pm 0.24$ & 0.724 \\
Total cholesterol, mg/dl & $225.0 \pm 38.0$ & $174.0 \pm 30.0$ & $<0.001^{\mathrm{a}}$ \\
LDL-cholesterol, mg/dl & $138.0 \pm 31.0$ & $72.0 \pm 15.0$ & $<0.00001^{\mathrm{a}}$ \\
HDL-cholesterol, mg/dl & $66.0 \pm 16.0$ & $71.0 \pm 23.0$ & 0.557 \\
TG, mg/dl & $121.0 \pm 41.0$ & $89.0 \pm 22.0$ & 0.237 \\
Non-HDL, mg/dl & $159.0 \pm 31.0$ & $103.0 \pm 14.0$ & $<0.0001^{\text {a }}$ \\
LDL/HDL-cholesterol ratio & $2.13 \pm 0.55$ & $1.30 \pm 0.39$ & $0.0002^{\mathrm{a}}$ \\
HDL/Total cholesterol, \% & $29.5 \pm 5.6$ & $40.0 \pm 7.9$ & $0.0005^{\mathrm{a}}$ \\
TG/HDL-cholesterol ratio & $1.61 \pm 0.81$ & $1.42 \pm 0.70$ & 0.537 \\
hsCRP, mg/l & $4.21 \pm 1.68$ & $3.66 \pm 1.22$ & 0.432 \\
HB, g/dl & $12.6 \pm 1.3$ & $12.3 \pm 1.30$ & 0.621 \\
\hline
\end{tabular}

${ }^{\mathrm{a}} \mathrm{P}<0.001 ;{ }^{\mathrm{b}}$ mean \pm standard deviation $\mathrm{D}$, dyslipidemia; HB, hemoglobin; HDL, high density lipoprotein; hsCRP, high sensitivity C reactive protein; LDL, low density lipoprotein; RAGE, receptor for advanced glycation end products; TG, triglyceride; VEGFR2, vascular endothelial growth factor receptor 2 .

Table V. Significant correlations between VEGFR2 and other clinicopathological characteristics in the examined groups treated with HD, with and without dyslipidemia.

A, Whole HD group, $n=50$

\begin{tabular}{lcc}
\hline Correlated parameter & $\mathrm{R}$ & P-value \\
\hline VEGFR2 and age & 0.334 & $0.017^{\mathrm{a}}$ \\
VEGFR2 and RAGE & 0.686 & $<0.00001^{\mathrm{c}}$ \\
VEGFR2 and Tchol & 0.297 & $0.036^{\mathrm{a}}$ \\
VEGFR2 and LDL-chol & 0.291 & $0.039^{\mathrm{a}}$ \\
VEGFR2 and TG & 0.362 & $0.009^{\mathrm{b}}$ \\
VEGFR2 and non-HDL & 0.361 & $0.009^{\mathrm{b}}$ \\
VEGFR2 and LDL/HDL-chol ratio & 0.402 & $0.004^{\mathrm{b}}$ \\
VEGFR2 and HDL/Tchol ratio & -0.403 & $0.004^{\mathrm{b}}$ \\
VEGFR2 and TG/HDL-chol ratio & 0.362 & $0.009^{\mathrm{b}}$ \\
\hline
\end{tabular}

B, Dyslipidemic HD group, $n=26$

\begin{tabular}{lll}
\hline Correlated parameter & $\mathrm{R}$ & P-value \\
\hline VEGFR2 and age & 0.397 & $0.04^{\mathrm{a}}$ \\
VEGFR2 and RAGE & 0.737 & $0.0002^{\mathrm{c}}$ \\
\hline
\end{tabular}

C, Non-dyslipidemic HD group, $n=24$

\begin{tabular}{lcl}
\hline Correlated parameter & $\mathrm{R}$ & P-value \\
\hline VEGFR2 and RAGE & 0.677 & $0.00027^{\mathrm{c}}$ \\
VEGFR2 and PLT & -0.457 & $0.024^{\mathrm{a}}$ \\
\hline
\end{tabular}

${ }^{\mathrm{a}} \mathrm{P}<0.05$; ${ }^{\mathrm{b}} \mathrm{P}<0.01$; ${ }^{\mathrm{c}} \mathrm{P}<0.001$. HDL-chol, high density lipoprotein cholesterol; LDL-chol, low density lipoprotein cholesterol; PLT, platelets; RAGE, receptor for advanced glycation end products; Tchol, total cholesterol; TG, triglyceride; VEGFR2, vascular endothelial growth factor receptor 2.
In the present study, lower levels of VEGFR2 were observed in patients on HD compared with the control subjects, which was in line with the results reported by Sepe et al (32). In their study on 26 patients receiving regular HD, the plasma VEGFR2 levels were also significantly lower compared with those in the 9 age-matched healthy volunteers, and were inversely correlated with serum homocysteine levels. The results of the present study suggested that the atherogenic components of blood lipids may be partly involved in the regulation of VEGFR2 expression. A significant correlation was identified between atherogenic components of the lipid profile and plasma VEGFR2 levels in patients on HD and controls. In the examined groups, no marked difference was identified between HD and controls regarding the percentage of subjects with D (52 vs. 57\%). Therefore, AI was assessed in these groups. Of note, $\leq 56 \%$ of patients on HD had atherogenic D, as calculated by AI. Conversely, there were no subjects with atherogenic D in the control group. In addition, a significant correlation was observed between the TG/HDL-chol ratio, which is a determinant of AI and plasma VEGFR2 levels in the HD group. This fact may suggest that AI may be more likely than D to affect plasma VEGFR2 levels in patients on HD.

There have been several reports of increased carbonyl stress (33), AGE production and RAGE expression (34) in patients with uraemia. In addition, Liu et al (20) demonstrated that AGEs and RAGE, whose activity in patients on HD was significantly elevated, may modulate VEGFR2 levels. It has been shown that the incubation of endothelial cells with methyloglyoxal, a major source of AGEs and peroxynitrate (ONOO-) production, modulates VEGFR2 protein levels through RAGE-mediated, ONOO-dependent and autophagy-induced VEGFR2 degradation on the cell membrane $(20,33,35)$. Furthermore, it has been shown that the formation of atherosclerotic lesions is accelerated in parallel with VEGFR2 and RAGE activity (21). The results of the present study were in line with those of these previous studies. In the present study, 
the plasma levels of VEGFR2 in patients on HD were positively correlated with plasma RAGE levels. Therefore, these mechanisms may be at least partly be involved in the modulation of plasma VEGFR2 levels in patients on HD.

$\mathrm{MeS}$ commonly occurs in patients on HD, and is closely associated with endothelial cell dysfunction and VEGFR2 expression (36-38). The association between plasma VEGFR2 levels and MeS was evaluated in patients on HD. No differences were observed in the plasma VEGFR2 levels between patients on HD with and without MeS. To the best of our knowledge, the present study is one of few studies to report an association between plasma VEGFR2 and MeS. The conflicting data reported to date may be due to differences in the measurements of sVEGFR2 $(37,38)$. In previous studies, compared with subjects with normal renal function, plasma or serum sVEGFR2 levels were significantly decreased (37) or increased (38) in subjects with MeS. In the present study, circulating plasma VEGFR2 (secreted and proteolytic shedded forms) were both measured, and no correlation was observed between MeS and circulating plasma VEGFR2 levels. Further studies are required to evaluate the association between the components of MeS and plasma VEGFR2 levels.

NAFLD is known to be strongly associated with MeS, and is considered as a novel risk factor of cardiovascular events in patients undergoing HD (38-40). Wu et al (41) reported that $24 \%$ of the examined patients on HD had NAFLD. In the present study, $20 \%$ of patients on HD were found to have NAFLD based on the ultrasonography examination. The association between NAFLD and circulating VEGFR2 levels was also examined, but no correlations were found, consistent with previous studies $(13,14)$.

$\mathrm{D}$ is the primary characteristic of $\mathrm{MeS}$ and a common feature amongst patients on HD. In the present study, $>50 \%$ of the patients on HD were also diagnosed with $\mathrm{D}$, based on the diagnostic recommendations of the K/DOQI guidelines (27). Hyper-LDL-cholesterolemia was also recorded in $91 \%$ of the subjects. In addition, in dyslipidemic patients on HD, serum TG was significantly higher compared with the non-dyslipidemic HD group. A growing body of evidence supports the notion that ESRD is associated with hypertriglyceridemia $(27,8,39,40)$. Recently, Vaziri et al (42) demonstrated that the novel endothelium-derived molecule glycosylphosphatidylinositol-anchored binding protein 1 (GPIHBP1) is involved in the development of hypertriglyceridemia in patients with chronic kidney disease. Other studies have found that the VEGF-VEGFR axis modulates GPIHBP1 mRNA and protein expression levels in endothelial cells (43). Consistent with these findings, the results of the present study support the hypothesis that VEGFR2 may be partially involved in the development of hypertriglyceridemia through the VEGF-VEGFR axis. Additionally, it was found that circulating VEGFR2 levels were positively correlated with TG in patients on HD and control subjects.

The association between LDL-chol and plasma VEGFR2 levels was also examined. Data from previous studies indicated that LDL-chol affects the structure and activity of VEGFR2, but the mechanism underlying this phenomenon is not fully understood. Jin et al (44) demonstrated that LDL-chol attenuates endothelial VEGFR2 expression. It was revealed that the loss of VEGFR2 resulted from its internalization and degradation in endosome-trans-Golgi network trafficking. Conversely, a study by Rodrígues et al (45) on an animal model revealed that native LDL-chol significantly upregulated VEGF and VEGFR2 in endothelial cells, and this upregulation was associated with an oxidative stress-mediated mechanism. In addition, it has been established that oxidized phospholipids may stimulate the expression of VEGFR2 (46). Since the oxidized LDL-chol that accumulates during atherosclerosis (47) contains large amounts of oxidized phospholipids, a connection between LDL-chol metabolism and VEGFR2 may be considered. In the present study, a positive correlation was identified between plasma circulating VEGFR2 levels and LDL-chol, non-HDL and the LDL/HDL-chol ratio, in agreement with previous studies $(45,46)$.

Recently, the HDL/Tchol ratio was reported to be a more reliable risk factor for atherosclerotic changes compared with Tchol or HDL-chol alone (48). Since a low HDL/Tchol ratio (47) and high endothelial expression of VEGFR2 (46) are considered risk factors for atherosclerosis, the association between HDL/Tchol ratio and plasma VEGFR2 levels was evaluated in the present study. A negative correlation was identified between plasma circulating VEGFR2 levels and the HDL/Tchol ratio. These findings support the notion that not only membranous VEGFR2, but also circulating VEGFR2 expression, may be partially modulated by lipid components.

The present study has several limitations. First, all patients in this study were Caucasian, and any differences with other ethnicities were not examined. Second, single-center trials are associated with a potential bias. Third, the number of patients and healthy subjects was relatively small.

In conclusion, the findings of the present study demonstrated that circulating VEGFR2 levels were lower in patients on HD compared with those in the healthy population, D was associated with higher plasma VEGFR2 levels, the TG/HDL-chol ratio (an index of atherogenic D) was strongly associated with plasma VEGFR2 levels, and plasma VEGFR2 concentration was associated with circulating RAGE levels. It may be inferred that, in patients on HD, circulating VEGFR2 levels may be partially associated with lipid abnormalities and plasma RAGE levels. However, further studies are required to define the pathophysiological role of circulating VEGFR2 and its precise association with $\mathrm{D}$.

\section{Acknowledgements}

Not applicable.

\section{Funding}

No funding was received.

\section{Availability of data and materials}

The datasets used and/or analyzed during the present study are available from the corresponding author on reasonable request.

\section{Authors' contributions}

LN conceived and designed the study, and was involved in data collection and analysis. HD performed the analyses of VEGFR2 and RAGE levels and data analysis. WW was 
involved in data analysis and performed the statistical analysis. All the authors have read and approved the final version of the manuscript. HD and WW confirm the authenticity of all the raw data.

\section{Ethics approval and consent to participate}

The protocol used in the present study was performed in accordance with the guidelines described in the Declaration of Helsinki and was approved by the Institutional Review Board of Poznań University of Medical Sciences, Poland. Written informed consent was obtained from all subjects before participation.

\section{Patient consent for publication}

Not applicable.

\section{Competing interests}

The authors declare that they have no competing interests.

\section{References}

1. Machnik A, Neuhofer W, Jantsch J, Dahlmann A, Tammela T, Machura K, Park JK, Beck FX, Müller DN, Derer W, et al: Macrophages regulate salt-dependent volume and blood pressure by a vascular endothelial growth factor-C-dependent buffering mechanism. Nat Med 15: 545-552, 2009.

2. Wheeler-Jones C, Abu-Ghazaleh R, Cospedal R, Houliston RA, Martin J and Zachary I: Vascular endothelial growth factor stimulates prostacyclin production and activation of cytosolic phospholipase A2 in endothelial cells via p42/p44 mitogen-activated protein kinase. FEBS Lett 420: 28-32, 1997.

3. Ferrara N: Vascular endothelial growth factor. Eur J Cancer 32A: 2413-2422, 1996.

4. Roskoski R Jr: VEGF receptor protein-tyrosine kinases: Structure and regulation. Biochem Biophys Res Commun 375: 287-291, 2008.

5. Rahimi N, Golde TE and Meyer RD: Identification of ligand-induced proteolytic cleavage and ectodomain shedding of VEGFR-1/FLT1 in leukemic cancer cells. Cancer Res 69: 2607-2614, 2009.

6. Ebos JM, Bocci G, Man S, Thorpe PE, Hicklin DJ, Zhou D, Jia X and Kerbel RS: A naturally occurring soluble form of vascular endothelial growth factor receptor 2 detected in mouse and human plasma. Mol Cancer Res 2: 315-326, 2004.

7. Koga K, Osuga Y, Yoshino O, Hirota Y, Ruimeng X, Hirata T, Takeda S, Yano T, Tsutsumi O and Taketani Y: Elevated serum soluble vascular endothelial growth factor receptor 1 (sVEGFR-1) levels in women with preeclampsia. J Clin Endocrinol Metab 88: 2348-2351, 2003.

8. Rath G and Tripathi R: VEGF and its soluble receptor VEGFR-2 in hypertensive disorders during pregnancy: The Indian scenario. J Hum Hypertens 26: 196-204, 2012.

9. Zachary I, Mathur A, Yla-Herttuala S and Martin J: Vascular protection: A novel nonangiogenic cardiovascular role for vascular endothelial growth factor. Arterioscler Thromb Vasc Biol 20: 1512-1520, 2000.

10. Servos S, Zachary I and Martin JF: VEGF modulates NO production: The basis of a cytoprotective effect? Cardiovasc Res 41: 509-510, 1999.

11. Yap RW, Shidoji Y, Hon WM and Masaki M: Association and interaction between dietary pattern and VEGF receptor-2 (VEGFR2) gene polymorphisms on blood lipids in Chinese Malaysian and Japanese adults. Asia Pac J Clin Nutr 21: 302-311, 2012.

12. Kubisz P, Chudý P, Stasko J, Galajda P, Hollý P, Vysehradský R and Mokán M: Circulating vascular endothelial growth factor in the normo- and/or microalbuminuric patients with type 2 diabetes mellitus. Acta Diabetol 47: 119-124, 2010.
13. Coulon S, Francque S, Colle I, Verrijken A, Blomme B Heindryckx F, De Munter S, Prawitt J, Caron S, Staels B, et al: Evaluation of inflammatory and angiogenic factors in patients with non-alcoholic fatty liver disease. Cytokine 59: 442-449, 2012.

14. Jaroszewicz J, Januszkiewicz M, Flisiak R, Rogalska M, Kalinowska A and Wierzbicka I: Circulating vascular endothelial growth factor and its soluble receptors in patients with liver cirrhosis: Possible association with hepatic function impairment. Cytokine 44: 14-17, 2008.

15. Kim BM, Lee DH, Choi HJ, Lee KH, Kang SJ, Joe YA, Hong YK and Hong SH: The recombinant kringle domain of urokinase plasminogen activator inhibits VEGF165-induced angiogenesis of HUVECs by suppressing VEGFR2 dimerization and subsequent signal transduction. IUBMB Life 64: 259-265, 2012.

16. Noghero A, Perino A, Seano G, Saglio E, Lo Sasso G, Veglio F, Primo L, Hirsch E, Bussolino F and Morello F: Liver X receptor activation reduces angiogenesis by impairing lipid raft localization and signaling of vascular endothelial growth factor receptor-2. Arterioscler Thromb Vasc Biol 32: 2280-2288, 2012.

17. Bodzioch M, Orsó E, Klucken J, Langmann T, Böttcher A, Diederich W, Drobnik W, Barlage S, Büchler C, PorschOzcürümez M, et al: The gene encoding ATP-binding cassette transporter 1 is mutated in Tangier disease. Nat Genet 22: 347-351, 1999.

18. Fang L, Choi SH, Baek JS, Liu C, Almazan F, Ulrich F, Wiesner P, Taleb A, Deer E, Pattison J, et al: Control of angiogenesis by AIBP-mediated cholesterol efflux. Nature 498: 118-122, 2013.

19. Zhang M and Jiang L: Oxidized low-density lipoprotein decreases VEGFR2 expression in HUVECs and impairs angiogenesis. Exp Ther Med 12: 3742-3748, 2016

20. Liu H, Yu S, Zhang $\mathrm{H}$ and $\mathrm{Xu}$ J: Angiogenesis impairment in diabetes: Role of methylglyoxal-induced receptor for advanced glycation endproducts, autophagy and vascular endothelial growth factor receptor 2. PLoS One 7: e46720, 2012.

21. Roy H, Bhardwaj S, Babu M, Kokina I, Uotila S, Ahtialansaari T, Laitinen T, Hakumaki J, Laakso M, Herzig KH, et al: VEGF-A, VEGF-D, VEGF receptor-1, VEGF receptor-2, NF-kappaB, and RAGE in atherosclerotic lesions of diabetic Watanabe heritable hyperlipidemic rabbits. FASEB J 20: 2159-2161, 2006.

22. World Medical Association Declaration of Helsinki: Ethical principles for medical research involving human subjects: JAMA 310: 2191-2194, 2013.

23. Update of the National Kidney Foundation-Kidney Disease Outcomes Quality Initiative Clinical Practice Guideline for Hemodialysis Adequacy. 2015. https://www.ajkd.org/article/S02726386(15)01019-7/fulltext.

24. Kouw PM, Olthof CG, ter Wee PM, Oe LP, Donker AJ, Schneider $\mathrm{H}$ and de Vries PM: Assessment of post-dialysis dry weight: An application of the conductivity measurement method. Kidney Int 41: 440-444, 1992.

25. Hamaguchi M, Kojima T, Itoh Y, Harano Y, Fujii K, Nakajima T, Kato T, Takeda N, Okuda J, Ida K, et al: The severity of ultrasonographic findings in nonalcoholic fatty liver disease reflects the metabolic syndrome and visceral fat accumulation. Am J Gastroenterol 102: 2708-2715, 2007.

26. Reiner Z, Catapano AL, De Backer G, Graham I, Taskinen MR, Wiklund O, Agewall S, Alegria E, Chapman MJ, Durrington P, et al; European Association for Cardiovascular Prevention and Rehabilitation; ESC Committee for Practice Guidelines (CPG) 2008-2010 and 2010-2012 Committees: ESC/EAS Guidelines for the management of dyslipidaemias: The Task Force for the management of dyslipidaemias of the European Society of Cardiology (ESC) and the European Atherosclerosis Society (EAS). Eur Heart J 32: 1769-1818, 2011.

27. Kidney Disease Outcomes Quality Initiative (K/DOQI) Group: K/DOQI clinical practice guidelines for management of dyslipidemias in patients with kidney disease. Am J Kidney Dis 41 (Suppl 3): I-IV, S1-S91, 2003.

28. Hanak V, Munoz J, Teague J Jr, Stanley A Jr and Bittner V: Accuracy of the triglyceride to high-density lipoprotein cholesterol ratio for prediction of the low-density lipoprotein phenotype B. Am J Cardiol 94: 219-222, 2004.

29. Friedewald WT, Levy RI and Fredrickson DS: Estimation of the concentration of low-density lipoprotein cholesterol in plasma, without use of the preparative ultracentrifuge. Clin Chem 18: 499-502, 1972 .

30. Matthews DR, Hosker JP, Rudenski AS, Naylor BA, Treacher DF and Turner RC: Homeostasis model assessment: Insulin resistance and beta-cell function from fasting plasma glucose and insulin concentrations in man. Diabetologia 28: 412-419, 1985. 
31. Alberti G, Shaw J and Zimmet P: A new IDF worldwide definition of the metabolic syndrome: The rationale and the results. Diabetes Voice 50: 31-33, 2005.

32. Sepe V, Libetta C, Rossi N, Guidetti C and Dal Canton A: Inverse association between homocysteine and vascular endothelial growth factor receptor 2 serum levels in hemodialyzed and kidney transplanted patients. Kidney Int 64: 1922, 2003.

33. Karg E, Papp F, Tassi N, Janáky T, Wittmann G and Túri S: Enhanced methylglyoxal formation in the erythrocytes of hemodialyzed patients. Metabolism 58: 976-982, 2009.

34. Stinghen AE, Massy ZA, Vlassara H, Striker GE and Boullier A: Uremic toxicity of advanced glycation end products in CKD. J Am Soc Nephrol 27: 354-370, 2016.

35. Schmidt S, Westhoff TH, Krauser P, Zidek W and van der Giet M: The uraemic toxin phenylacetic acid increases the formation of reactive oxygen species in vascular smooth muscle cells. Nephrol Dial Transplant 23: 65-71, 2008.

36. Mahdy RA, Nada WM, Hadhoud KM and El-Tarhony SA: The role of vascular endothelial growth factor in the progression of diabetic vascular complications. Eye (Lond) 24: 1576-1584, 2010.

37. Jesmin S, Akter S, Rahman MM, Islam MM, Islam AM, Sultana SN, Mowa CN, Yamaguchi N, Okazaki O, Satoru K, et al: Disruption of components of vascular endothelial growth factor angiogenic signalling system in metabolic syndrome. Findings from a study conducted in rural Bangladeshi women. Thromb Haemost 109: 696-705, 2013.

38. Wada H, Satoh N, Kitaoka S, Ono K, Morimoto T, Kawamura T, Nakano T, Fujita M, Kita T, Shimatsu A, et al: Soluble VEGF receptor-2 is increased in sera of subjects with metabolic syndrome in association with insulin resistance. Atherosclerosis 208 $512-517,2010$

39. Lai YC, Cheng BC, Hwang JC, Lee YT, Chiu CH, Kuo LC and Chen JB: Association of fatty liver disease with nonfatal cardiovascular events in patients undergoing maintenance hemodialysis. Nephron Clin Pract 124: 218-223, 2013.

40. Capone D, Vinciguerra M, Ragosta A, Citro V and Tarantino G: Troponin levels relate to CRP concentrations in patients with NAFLD on maintenance Haemodialysis: A retrospective study. Adv Ther 37: 3337-3347, 2020.
41. Wu PJ, Chen JB, Lee WC, Ng HY, Lien SC, Tsai PY, Wu CH, Lee CT and Chiou TT: Oxidative stress and nonalcoholic fatty liver disease in hemodialysis patients. BioMed Res Int 2018: 3961748,2018

42. Vaziri ND, Yuan J, Ni Z, Nicholas SB and Norris KC: Lipoprotein lipase deficiency in chronic kidney disease is accompanied by down-regulation of endothelial GPIHBP1 expression. Clin Exp Nephrol 16: 238-243, 2012

43. Chiu AP, Wan A, Lal N, Zhang D, Wang F, Vlodavsky I, Hussein B and Rodrigues B: Cardiomyocyte VEGF regulates endothelial cell GPIHBP1 to relocate lipoprotein lipase to the coronary lumen during diabetes mellitus. Arterioscler Thromb Vasc Biol 36: 145-155, 2016.

44. Jin F, Hagemann N, Brockmeier U, Schäfer ST, Zechariah A and Hermann DM: LDL attenuates VEGF-induced angiogenesis via mechanisms involving VEGFR2 internalization and degradation following endosome-trans-Golgi network trafficking. Angiogenesis 16: 625-637, 2013.

45. Rodríguez JA, Nespereira B, Pérez-Ilzarbe M, Eguinoa E and Páramo JA: Vitamins C and E prevent endothelial VEGF and VEGFR-2 overexpression induced by porcine hypercholesterolemic LDL. Cardiovasc Res 65: 665-673, 2005.

46. Zimman A, Mouillesseaux KP, Le T, Gharavi NM, Ryvkin A, Graeber TG, Chen TT, Watson AD and Berliner JA: Vascular endothelial growth factor receptor 2 plays a role in the activation of aortic endothelial cells by oxidized phospholipids. Arterioscler Thromb Vasc Biol 27: 332-338, 2007.

47. Ylä-Herttuala S, Palinski W, Rosenfeld ME, Parthasarathy S, Carew TE, Butler S, Witztum JL and Steinberg D: Evidence for the presence of oxidatively modified low density lipoprotein in atherosclerotic lesions of rabbit and man. J Clin Invest 84: 1086-1095, 1989.

48. van Merode T, Hick P, Hoeks PG and Reneman RS: Serum $\mathrm{HDL} /$ total cholesterol ratio and blood pressure in asymptomatic atherosclerotic lesions of the cervical carotid arteries in men. Stroke 16: 34-38, 1985.

This work is licensed under a Creative Commons Attribution-NonCommercial-NoDerivatives 4.0 International (CC BY-NC-ND 4.0) License. 